\title{
A New Geometrical Approach to Solve Inverse kinematics of Hyper Redundant Robots with variable link length
}

\author{
Annisa Jamali, Raisuddin Khan and Md. Mozasser Rahman \\ Department of Mechatronics Engineering, Faculty of Engineering \\ International Islamic University Malaysia, Jalan Gombak, 53100 Kuala Lumpur, Malaysia \\ raisuddin@iium.edu.my
}

\begin{abstract}
In this paper a new approach that generates a general algorithm for n-link hyper-redundant robot is presented. This method uses repetitively the basic inverse kinematics solution of a 2- link robot on some virtual links, where the virtual links are defined following some geometric proposition. Thus, it eliminates the mathematical complexity in computing inverse kinematics solution of n-link hyper redundant robot. Further, this approach can handle planar manipulator with variable links eliminating singularity. Numerical simulations for planar hyper redundant models are presented in order to illustrate the competency of the model.
\end{abstract}

Keywords- Robot; Hyper-redundant; Variable length links; Inverse kinematics.

\section{INTRODUCTION}

Technology evolvements arise in parallel to the increasing number of robotics application. A robot having more degrees of freedom (DOF) than are necessary to perform a specific task is referred to as kinematically redundant or hyperredundant robot [1]. Even though existence of redundancy complicates the solution process, redundant degree of freedom has been recognized as a mean to improve manipulator performance in complex and unstructured environments tremendously. Idea of hyper redundant manipulator is promising due to their ability and applicability in many complicated scenario such as avoidance of obstacles, easy access into cluttered and inaccessible workspace, reconfigurable in the case of failure of few joints, etc. Thus, generating algorithm for hyper-redundant manipulator to handle aforementioned complex scenario is one of the interests of the today's researchers.

In conventional robot control theory, the manipulator Jacobian is considered as one of the most important tools [2]. Earlier in 1980's, research on inverse kinematics problem of hyper redundant robot was focused on Jacobian pseudo inverse approach and it's optimization [2][3]. At present, there are numbers of algorithms for the redundant manipulator inverse kinematics. The algorithm formulation can be divided mainly into three categories [4] that is (i) the algebraic approach [5]. (ii) the iterative approach that include Artificial
Neural Network [6], genetics algorithm , ANFIS [7], Fuzzy Logic [8] and (iii) the geometric approach [9][10][11][12][13].

In general, algebraic approach suffers from heavy computational burden and difficult symbolic expansion. Besides, although this approach offers several possible solutions with different arm configuration, but there is no proper indication to choose the best solution. Meanwhile, iterative approach involves high time-consuming computation where it takes considerable time to come up with useful inverse kinematics (IK) solution. Moreover, error minimizing always becomes a subject of investigation which also due to several possible solutions offered by this approach. The geometric approach means finding $\theta_{i}$ using geometrical heuristics to take advantage of the special structure of the manipulator. Thus, geometrical approach offers a direct solution.

This paper proposes a new geometrical approach to solve inverse kinematics of hyper redundant manipulator, where the length of the manipulator is adjustable. The method involves repetitive use of inverse kinematics solution of 2-link manipulator based on some geometric proposition, which depends on the selection of some reference point and virtual configuration. A coil shape is then formed out of the serially connected n-link which allows the system to avoid singularity. The paper is organized as follows; section 2 presents the details of algorithm formulation; section 3 verifies by computer simulation the general algorithm; and section 4 draws conclusions based on the performance of the algorithm.

\section{DETAILS OF ALGORITHM FormULATION}

\section{A. Defining Virtual Layers}

At the beginning, a 2-link virtual robot is introduced by taking length of the virtual links equal to the sum of lengths of half of the total number of links, $n$. However, in the case of $n$ an odd number, the virtual link closer to the origin will have even number of links and the other virtual link will have odd number of links. This 2-link virtual robot is then solved for inverse kinematics with either elbow-up or elbow-down configuration, so that the tip of the robotic manipulator reaches the desired position. This makes the $1^{\text {st }}$ virtual layer $\mathrm{SR}_{1}$. In Fig. $1 \mathrm{OO}_{1} \mathrm{P}$ is the virtual layer $\mathrm{SR}_{1}$ formed out of an 8 -link robotic manipulator, where $\mathrm{P}$ is the desired position and $\mathrm{O}$ is the origin. In the next step each of the virtual links of $\mathrm{SR}_{1}$ is

The authors would like to thank Research Management Centre, International Islamic University Malaysia for funding this research. 